\title{
Effect of Plant Characteristics and Within-Plant Distribution of Prey on Colonization Efficiency of Cryptolaemus montrouzieri (Coleoptera: Coccinellidae) Adults
}

\author{
Folukemi Adedipe and Yong-Lak Park \\ Department of Entomology, West Virginia University, Morgantown, WV, USA \\ Correspondence should be addressed to Yong-Lak Park, yopark@mail.wvu.edu \\ Received 2 October 2011; Accepted 20 December 2011 \\ Academic Editor: Martin H. Villet
}

Copyright ( $) 2012$ F. Adedipe and Y.-L. Park. This is an open access article distributed under the Creative Commons Attribution License, which permits unrestricted use, distribution, and reproduction in any medium, provided the original work is properly cited.

\begin{abstract}
Cryptolaemus montrouzieri (Coleoptera: Coccinellidae) has been widely used in classical and inundative biological control of mealybugs, including the long-tailed mealybug, Pseudococcus longispinus (Hemiptera: Pseudococcidae). This study was conducted to investigate colonization and establishment efficiency of $C$. montrouzieri to manage P. longispinus on three different ornamental plant species (Ficus elastica, Lilium longiflorum, and Dieffenbachia seguine). Within-plant distribution pattern of $P$. longispinus and the colonization ecology of adult C. montrouzieri were investigated. Significantly more P. longispinus were found on the upper parts of the plants regardless of plant species, and C. montrouzieri adults discovered P. longispinus significantly faster when they were released on the top of the plants than on the bottom. Choice tests revealed that $C$. montrouzieri adults preferred smaller $P$. longispinus nymphs. The implications for utilization of $C$. montrouzieri for biological control of mealybugs on various ornamental plants are discussed.
\end{abstract}

\section{Introduction}

The long-tailed mealybug, Pseudococcus longispinus (Targioni-Tozzetti) (Hemiptera: Pseudococcidae), is a key pest of fruit trees and ornamental plants. P. longispinus feeds on various plant parts including roots, trunks, cordons, canes, leaves, and fruits, causing aesthetic damage on ornamental plants or yield loss of crops [1]. Fungal pathogens that grow on the honeydew excreted by $P$. longispinus can cause further damage. For example, high $P$. longispinus densities often cause leaf drop and reductions of crop quality and yield; Uygun [2] reported that yield loss of citrus due to $P$. longispinus could be up to $80-90 \%$. Also, P. longispinus can transmit viral diseases in grapevines [3]. Chemical management of $P$. longispinus is difficult because it produces thick layers of protective wax and can hide in bark crevices, spurs, or canes. In general, chemical control is only effective when $P$. longispinosus is in the crawler stage and when host plants do not afford physical refuges from chemical sprays
[4]. Therefore, biological control using natural enemies has been a major alternate method to manage $P$. longispinus [5].

Natural enemies utilized to manage $P$. longispinus include lady beetles, parasitic wasps, and lacewings [6]. Among the natural enemies, the mealybug destroyer, Cryptolaemus montrouzieri Mulsant (Coleoptera: Coccinellidae), is one of the key natural enemies of $P$. longispinus. $C$. montrouzieri is native to Australia and has been introduced to manage many mealybug species throughout the world $[7,8]$. In the United States, C. montrouzieri was first imported in the late 1800 s to manage mealybugs in California [9]. Since then, welldefined and efficient rearing techniques were developed [10], and thus $C$. montrouzieri has been commercially available to growers throughout the United States.

Cryptolaemus montrouzieri has been used for different biological applications: classical biological control [11] and augmentative biological control $[12,13]$. In an established population, immature stages of $C$. montrouzieri dominate the stable age distribution, and most prey is consumed by 
the larvae [14]. However, adult stage of C. montrouzieri is released when biological control of $P$. longispinus is initiated because of their ability to disperse and colonize. Also, it is assumed that adults will lay eggs in suitable locations and give rise to another generation that provides the majority of pest suppression when they are in the larval stage.

Effectiveness of natural enemies is dependent upon the ability of the organism to establish populations in a given environment and find prey rapidly [15]. Previous studies showed that natural enemies' ability to establish and search for prey was affected by plant structure and size [16-20]. Garcia and O'Neil [15] showed that plant size and variegation affected the searching efficiency of $C$. montrouzieri, and Merlin et al. [21] found that oviposition of C. montrouzieri was stimulated by wax filaments produced by its prey. Also, these studies indicated that successful biological control of $P$. longispinus would be affected by how efficiently newly released C. montrouzieri adults search for P. longispinus. Specifically, there is a high chance for C. montrouzieri adults to successfully establish when they can start to search and find the suitable prey as soon as they are released. In addition, prey-size choice could affect successful colonization of predators [22]. Therefore, key factors influencing prey search efficiency of $C$. montrouzieri may include release location of C. montrouzieri based on within-plant distribution of $P$. longispinus, plant characteristics, and stages of $P$. longispinus that C. montrouzieri adults prefer.

This study was conducted to investigate colonization efficiency of C. montrouzieri to manage P. longispinus on three different types of ornamental plants. The objectives of this study were (1) to investigate within-plant distribution of $P$. longispinus, (2) to quantify the searching time of $C$. montrouzieri related to release location, and (3) to determine preference of $C$. montrouzieri adults for the size of $P$. longispinus.

\section{Materials and Methods}

All experiments were conducted in the greenhouse and the entomology laboratory of West Virginia University, Morgantown, WV, U.S.A.

2.1. Within-Plant Distribution of P. longispinus. We obtained three common species of ornamental plants from the greenhouse at West Virginia University (Monongalia County, WV, USA). The ornamental plants in this study include Ficus elastica (Urticales: Moraceae) $(86-94 \mathrm{~cm}$ in height), Lilium longiflorum (Liliales: Liliaceae) $(42-61 \mathrm{~cm}$ in height), and Dieffenbachia seguine (Alismatales: Araceae) $(23-27 \mathrm{~cm}$ in height). These plants were selected because they are very common ornamental plants produced in the greenhouse. These plants had been infested with $P$. longispinus for at least one year before experiments to obtain moderate-tohigh density of $P$. longispinus. Five plants of each plant species with similar infestation levels were selected, and the total numbers of $P$. longispinus nymphs and adults were counted on the upper and lower halves of each plant. Densities of
P. longispinus on upper and lower parts of each plant species were compared using two-way ANOVA at 5\% error rate [23].

2.2. Prey Searching Time of C. montrouzieri Adults on Three Different Plant Species. C. montrouzieri adults were maintained in ventilated cages with $P$. longispinus and a honey-water solution under laboratory conditions of $25^{\circ} \mathrm{C}$ and $16: 8(\mathrm{~L}: \mathrm{D}) \mathrm{h}$ photoperiod. C. montrouzieri were reared on P. longispinus. All C. montrouzieri adults were denied prey but had access to water for the $12 \mathrm{~h}$ period preceding all experiments. One randomly chosen $C$. montrouzieri adult was introduced onto either top (i.e., top shoot) or bottom (i.e., bottom part of stem within $2 \mathrm{~cm}$ above the soil line) of a plant. Once one C. montrouzieri adult was introduced to a plant, searching time of $C$. montrouzieri was measured. Searching time was measured as the duration between introduction and finding the first prey. This experiment was replicated five times for each plant species and each release location. Each C. montrouzieri adult was used for the test only once. The searching time of C. montrouzieri was recorded and analyzed with two-way (i.e., releasing locations and plant species) ANOVA at 5\% error rate (SAS Institute, 2008).

Because we used the same plants for repeated release of different C. montrouzieri adults in the experiment, any leftover chemical cues by previously used C. montrouzieri adults could affect the next adults introduced to the plant. Therefore, we examined the effect of leftover chemical cues by previously used $C$. montrouzieri adults on the next adults introduced to the plant by dividing the data into two groups: first ten and last ten introductions of $C$. montrouzieri. The searching time of the two groups was compared ANOVA at $5 \%$ error rate [23].

2.3. C. montrouzieri Preference to Prey Body Size. A total of 20 C. montrouzieri adults were denied prey but had access to plain water for the $12 \mathrm{~h}$ period preceding the experiment. Preference of $C$. montrouzieri for three different sizes of $P$. longispinus $(0.3 \pm 0.07,1.3 \pm 0.10$, and $3.0 \pm 0.14 \mathrm{~mm})$ was investigated using an empty 9-cm-diameter petri dish (LABTEK Division Miles Laboratories, Inc., Naperville, IL, USA) containing an excised leaf of $F$. elastic on the bottom of the Petri dish. Three P. longispinus with different body sizes were randomly placed on the leaf for each replication. One C. montrouzieri adult was placed in the center of the Petri dish and allowed to search for P. longispinus. C. montrouzieri adults' choice among the three different sizes of $P$. longispinus and handling and cleaning time was recorded. Handling time was measured from the start of first contact of $P$. longispinus by $C$. montrouzieri to cessation and included feeding. Cleaning time was measured as duration of waxy residue removal from the body. This choice test was run until the first nymph was consumed and replicated 20 times. Searching, handling, and cleaning times were compared using ANOVA at 5\% error rate [23], and the first choices by C. montrouzieri adults to feed on the three different body sizes of $P$. longispinus were compared using Chi-square test [24]. 
TABle 1: Mean $( \pm \mathrm{SD})$ number of $P$. longispinus on the upper and lower parts of three different plants. Note that there were no significant differences within columns.

\begin{tabular}{lcc}
\hline Plant species & Upper part of plant & Lower part of plant \\
\hline Ficus elastica & $415 \pm 264.5 \mathrm{a}^{*}$ & $110 \pm 93.2 \mathrm{~b}$ \\
Lilium longiflorum & $564 \pm 172.4 \mathrm{a}$ & $51 \pm 26.8 \mathrm{~b}$ \\
Dieffenbachia seguine & $441 \pm 154.1 \mathrm{a}$ & $51 \pm 40.4 \mathrm{~b}$ \\
\hline
\end{tabular}

* Means within rows followed by the same letter are not significantly different $(F$ test, $P<0.05)$.

\section{Results}

3.1. Within-Plant Distribution of P. longispinus. There were no significant differences in the total number of $P$. longispinus among the plants used in this study $(F=1.21 ; \mathrm{df}=2,57 ; P>$ $0.05)$. However, significantly $(F=57.4 ; \mathrm{df}=1,29 ; P<0.0001)$ more $P$. longispinus were found on the upper parts of the plants regardless of the plant species (Table 1). There were no significant differences in $P$. longispinus densities among the three plant species when $P$. longispinus densities on the upper and lower parts were compared: upper parts of plants $(F=0.46 ; \mathrm{df}=2,14 ; P=0.650)$ and the lower part of plants $(F=0.94 ; \mathrm{df}=2,14 ; P=0.443)$.

3.2. Prey Searching Time of C. montrouzieri Adults on Three Different Plant Species. The time C. montrouzieri spent to find the first $P$. longispinus was significantly different among the three different plant species $(F=7.9 ; \mathrm{df}=2,24 ; P=0.002)$ and the two different release points (top and bottom) $(F=$ 29.73; df $=1,24 ; P<0.001)$. Because interactions between plant species and release points $(F=7.37$; $\mathrm{df}=2,24 ; P<$ 0.001 ) were significant, we separately compared the time $C$. montrouzieri spent to find the first $P$. longispinus between two release locations for each plant height category. We found that C. montrouzieri spent significantly more time searching for $P$. longispinus when they were released from the bottom of the plants regardless of plant species (Figure 1). This indicates that prey-searching time for $C$. montrouzieri adults to find the first $P$. longispinus can be reduced by releasing them from the top of the plant.

We found that there were no differences in the searching $(F=1.93 ; \mathrm{df}=1,18 ; P>0.05)$, handling, and cleaning time $(F=1.21$; df $=1,58 ; P>0.05)$ between the first ten $C$. montrouzieri introduced to the plants and the next ten introduced. This indicates that there were no significant effects of chemical cues left, if any, on the searching behavior of C. montrouzieri adults in this study.

3.3. C. montrouzieri Preference to the Body Size of P. longispinus. The results of the preference test of $C$. montrouzieri adults for three different sizes of $P$. longispinus showed that there was significant $\left(\chi^{2}=9.109 ; \mathrm{df}=2 ; P<0.05\right)$ preference of C. montrouzieri adults for smaller P. longispinus compared to medium $\left(\chi^{2}=7.619 ; \mathrm{df}=1 ; P<0.01\right)$ and larger $\left(\chi^{2}=8.314 ; \mathrm{df}=1 ; P<0.01\right)$ sizes. Although there were no significant differences in handling time of $C$. montrouzieri

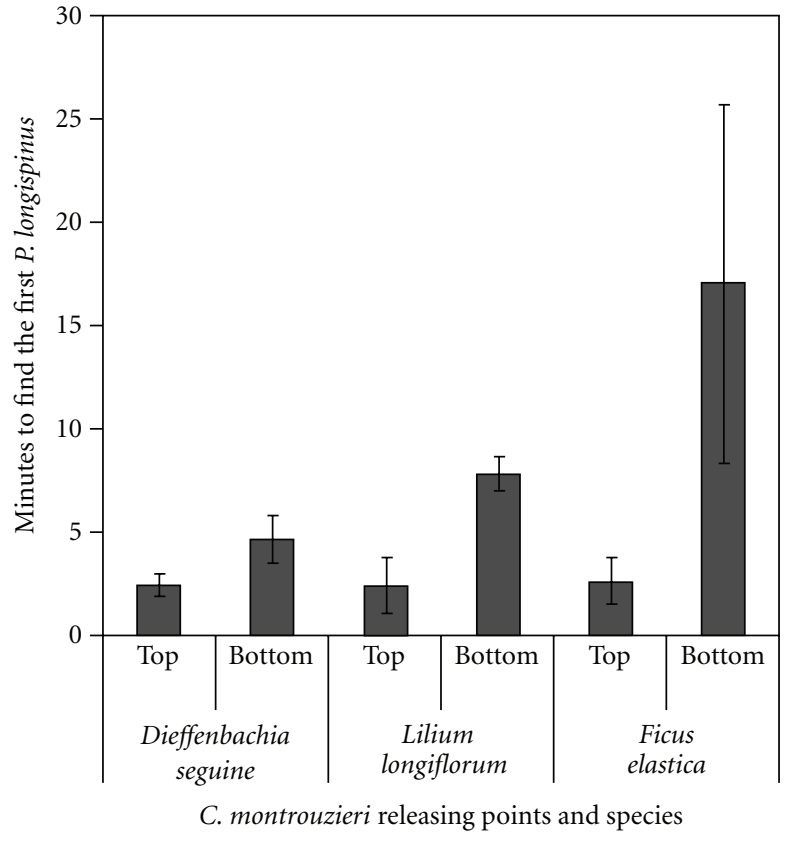

Figure 1: Effects of C. montrouzieri release locations and plant species on the time $C$. montrouzieri spent to find the first $P$. longispinus. Note that there were significant differences ( $F$ test; $P<$ 0.05 ) between two release locations regardless of plant species.

TABle 2: Handling and cleaning time (minutes \pm SD) of $C$. montrouzieri adults feeding on different sizes of mealybug.

\begin{tabular}{lccc}
\hline $\begin{array}{l}\text { Mealybug size } \\
(\text { mean } \pm \text { SD })\end{array}$ & Handling time & Cleaning Time & Total \\
\hline $\begin{array}{l}\text { Small } \\
(0.3 \pm 0.07 \mathrm{~mm})\end{array}$ & $6.9 \pm 2.18 \mathrm{a}^{*}$ & $3.5 \pm 2.03 \mathrm{~b}$ & $10.4 \pm 4.21 \mathrm{~b}$ \\
$\begin{array}{l}\text { Medium } \\
(1.3 \pm 0.10 \mathrm{~mm})\end{array}$ & $10.3 \pm 4.35 \mathrm{a}$ & $4.5 \pm 2.08 \mathrm{~b}$ & $14.8 \pm 6.43 \mathrm{ab}$ \\
$\begin{array}{l}\text { Large } \\
(3.0 \pm 0.14 \mathrm{~mm})\end{array}$ & $12.0 \pm 7.00 \mathrm{a}$ & $8.7 \pm 1.53 \mathrm{a}$ & $20.7 \pm 8.53 \mathrm{a}$ \\
\hline
\end{tabular}

* Means within columns followed by the same letter are not significantly different $(F$ test, $P<0.05)$.

feeding on different sizes of $P$. longispinus $(F=3.25 ; \mathrm{df}=2,17$; $P=0.064)$, there were significant differences in cleaning time among $C$. montrouzieri feeding on different sizes of $P$. longispinus ( $F=8.14 ; \mathrm{df}=2,17 ; P=0.003$ ) (Table 2). This result indicates that $C$. montrouzieri adults choose smaller $P$. longispinus more frequently and spend significantly more time to clean after feeding on larger P. longispinus.

\section{Discussion}

Within-plant distribution of pests is key information for determining where to release natural enemies. To maximize efficiency of biological control, prey-searching time could be reduced depending on where natural enemies are released $[18,25]$. In this study, we observed that significantly more $P$. longispinus inhabited the upper part of plants regardless 
of plant species. This observation is in agreement with the finding by Flaherty et al. [1] who showed higher population and movement of mealybugs toward the top of grapevines. Because C. montrouzieri is known to be more effective when $P$. longispinus populations are high $[8,26]$, releasing $C$. montrouzieri adults from the top of the plants could reduce prey-searching time because $P$. longispinus is abundant on the upper part of plants. In addition, plant species could affect prey-searching efficiency by natural enemies [15]. The results of our study indicated that effectiveness and establishment of C. montrouzieri increased when they were released on Dieffenbachia seguine, the smallest plant tested in this study. Therefore, the effectiveness of $C$. montrouzieri managing $P$. longispinus could be maximized when $C$. montrouzieri are released from the top and on smaller plants.

This study demonstrated that C. montrouzieri chose to feed on smaller P. longispinus. When $C$. montrouzieri fed on larger nymphs, they spent longer time handling and cleaning after feeding and before searching for another prey. This result is congruent with Merlin et al. [21] who found that C. montrouzieri consumed smaller P. longispinus nymphs first and then fed on larger nymphs or adults. Because establishment of natural enemies after release determines the success of augmentational biological control (i.e., inoculative release), finding the first prey by the natural enemies could increase the chance of establishment. Although a difference of 5-15 minutes in time to initial prey encounter may not make much of a difference to the final biological control outcome in a greenhouse, it could influence the chance of establishment because we frequently observed that $C$. montrouzieri adults could fly away to escape from the greenhouse when they cannot find the first prey in a reasonable period. Therefore, C. montrouzieri adults have higher chance to establish when the period for finding the first prey is shorter.

The results of this study suggest a major consideration for the use of $C$. montrouzieri to manage P. longispinus. The efficiency of $P$. longispinus management by $C$. montrouzieri depends on the location of $C$. montrouzieri release and the plant species. Our study showed that effectiveness and establishment of C. montrouzieri managing P. longispinus could be maximized when $C$. montrouzieri were released from the top of the plants and on the smaller plants, $C$. montrouzieri adults can reduce prey search time when they are released where $P$. longispinus is abundant. Future study needs to investigate the effect of plant age and stage on vertical distribution of $P$. longispinus on the plant.

\section{Acknowledgments}

The authors thank Vicki Kondo and JB White at West Virginia University for their valuable support and suggestions related to this research. They also thank Sue Myers at West Virginia University for her help in identifying plant species used in this research. This project was partially supported by the Division of Plant and Soil Science and the Faculty Senate Grant at West Virginia University.

\section{References}

[1] D. L. Flaherty, L. Christiensen, and W. T. Lanini, "Mealybugs," in Grape Pest Management, D. L. Flaherty, L. P. Christensen, W. T. Lanini, J. J. Marois, P. A. Phillips, and L. T. Wilson, Eds., pp. 159-165, University of California Division of Agriculture and Natural Resources Publication 3343, Calif, USA, 1992.

[2] N. Uygun, Integrated Pest Management in Turkish Citrus Orchard, Agricultural Research Project Publications, Adana, Turkey, 2001.

[3] H. Acheche, S. Fattouch, S. M'Hirsi, N. Marzouki, and M. Marrakchi, "Use of optimized PCR methods for the detection of GLRaV3: a closterovirus associated with grapevine leafroll in Tunisian grapevine plants," Plant Molecular Biology Reporter, vol. 17, no. 1, pp. 31-42, 1999.

[4] G. O. Furness, "Chemical and integrated control of the longtailed mealybug, Pseudococcus longispinus (Targioni-Tozzetti) (Hemiptera: Coccidae) in the Riverland of South Australia," Australian Journal of Agricultural Research, vol. 28, pp. 319332, 1977.

[5] S. Cooper, "Cryptolaemus montrouzieri: a predator for mealybug," British Cactus and Succulent Journal, vol. 3, pp. 38-39, 1985.

[6] E. Swirski, Y. Izhar, M. Wysoki, E. Gurevitz, and S. Greenberg, "Integrated control of the long-tailed mealybug, Pseudococcus longispinus [Hom.: Pseudococcidae], in avocado plantations in Israel," Entomophaga, vol. 25, no. 4, pp. 415-426, 1980.

[7] B. R. Bartlett, "Introduction into California of cold-tolerant biotypes of the mealybug predator, Cryptolaemus montrouzieri, and laboratory procedures for testing natural enemies for cold-hardness," Environmental Entomology, vol. 3, pp. 553-556, 1974.

[8] B. R. Bartlett, "Diaspididae, eriococcidae, margarodidae, ortheziidae, pseudococcidae," in Introduced Parasite and Predators of Arthropod Pests and Weeds: a World Review, P. Clausen C., Ed., U.S.D.A. Agriculture Handbook no. 480, pp. 57-170, Washington, DC, USA, 1978.

[9] C. Sadof, "Know your friends: mealybug destroyer," 1995, http://www.oisat.org/control_methods/natural_enemies/predators/mealybug_destroyer.html.

[10] J. C. van Lenteren, Quality Control and Production of Biological Control Agents: Theory and Testing Procedures, CABI Publishing, London, UK, 2003.

[11] M. M. Kinawy, H. M. Al-Waili, and A. M. Almandhari, "Review of the successful classical biological control programs in Sultanate of Oman," Egyptian Journal of Biological Pest Control, vol. 18, no. 1, pp. 1-10, 2008.

[12] S. F. Smith and V. A. Krischik, "Effects of biorational pesticides on four coccinellid species (Coleoptera: Coccinellidae) having potential as biological control agents in interiorscapes," Journal of Economic Entomology, vol. 93, no. 3, pp. 732-736, 2000.

[13] N. Al-Khateeb and L. Asslan, "Preliminary data on settlement and adaptation of the predator, Cryptolaemus montrouzieri mulsant (Coleoptera: Coccinellidae) in colonies of Mealy Bugs on citrus trees in the Syrian Coast," Egyptian Journal of Biological Pest Control, vol. 19, no. 2, pp. 191-192, 2009.

[14] A. Persad and A. Khan, "Comparison of life table parameters for Maconellicoccus hirsutus, Anagyrus kamali, Cryptolaemus montrouzieri and Scymnus coccivora," BioControl, vol. 47, no. 2, pp. 137-149, 2002.

[15] J. F. Garcia and R. J. O’Neil, “Effect of Coleus size and variegation on attack rates, searching strategy, and selected life history characteristics of Cryptolaemus montrouzieri (Coleoptera: 
Coccinellidae)," Biological Control, vol. 18, no. 3, pp. 225-234, 2000.

[16] P. W. Price, C. E. Bouton, P. Gross, B. A. McPheron, J. N. Thompson, and A. E. Weis, "Interactions among three trophic levels: Influence of plants on interactions between insect herbivores and natural enemies," Annual Review of Ecology and Systematics, vol. 11, pp. 41-65, 1980.

[17] J. H. Lawton, "Plant architecture and the diversity of phytophagous insects," Annual review of entomology, vol. 28, pp. 23-39, 1983.

[18] D. A. Andow and D. R. Prokrym, "Plant structural complexity and host-finding by a parasitoid," Oecologia, vol. 82, no. 2, pp. $162-165,1990$.

[19] P. Kareiva and R. Sahakian, "Tritrophic effects of a simple architectural mutation in pea plants," Nature, vol. 345, no. 6274, pp. 433-434, 1990.

[20] F. S. Grevstad and B. W. Klepetka, "The influence of plant architecture on the foraging efficiencies of a suite of ladybird beetles feeding on aphids," Oecologia, vol. 92, no. 3, pp. 399404, 1992.

[21] J. Merlin, O. Lemaitre, and J. C. Grégoire, "Oviposition in Cryptolaemus montrouzieri stimulated by wax filaments of its prey," Entomologia Experimentalis et Applicata, vol. 79, no. 2, pp. 141-146, 1996.

[22] G. Ernsting and D. C. Van Der Werf, "Hunger, partial consumption of prey and prey size preference in a carabid beetle," Ecological Entomology, vol. 13, no. 2, pp. 155-164, 1988.

[23] SAS Institute, SAS OnlineDoc Version 9.1.3, SAS Institute, Cary, NC, USA, 2008.

[24] R. R. Sokal and F. J. Rohlf, Biometry, W. H. Freeman and Company, New York, NY, USA, 1998.

[25] Y. Jinsong and C. S. Sadof, "Variegation in Coleus blumei and the life history of citrus mealybug (Homoptera: Pseudococcidae)," Environmental Entomology, vol. 24, no. 6, pp. 16501655, 1995.

[26] D. Moore, "Agents used for biological control of mealybugs (Pseudococcidae)," Biocontrol News and Information, vol. 9, pp. 209-225, 1988. 

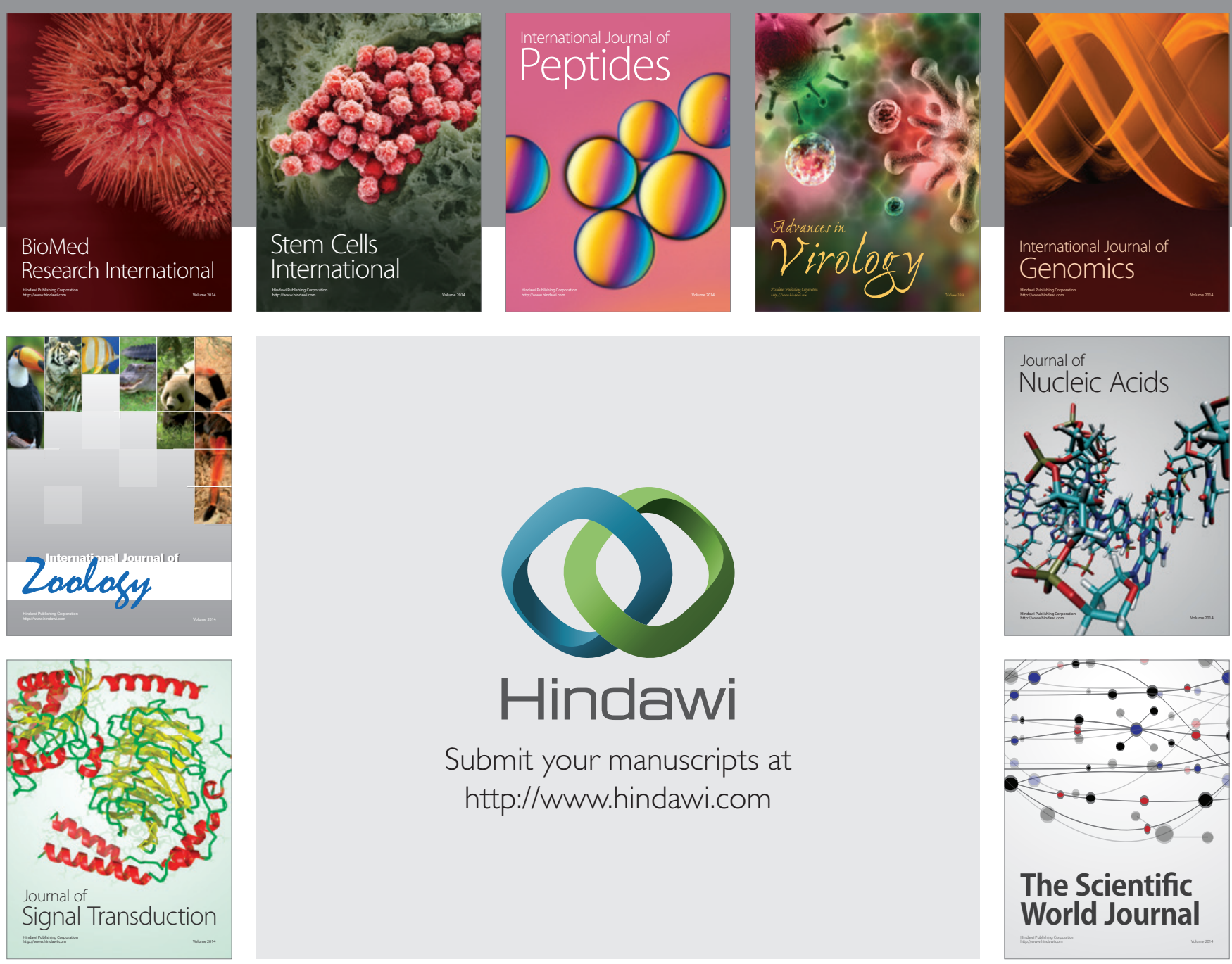

Submit your manuscripts at

http://www.hindawi.com
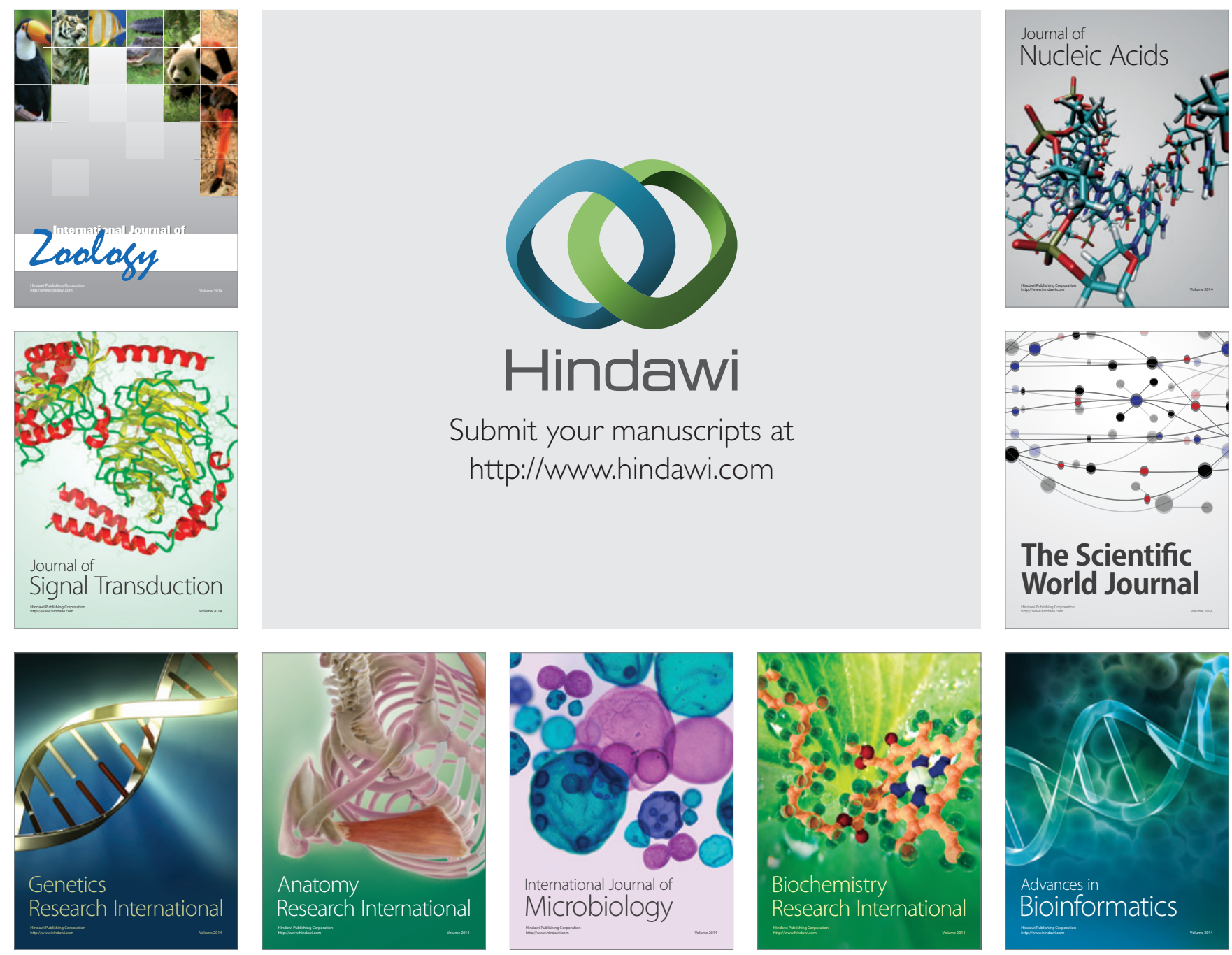

The Scientific World Journal
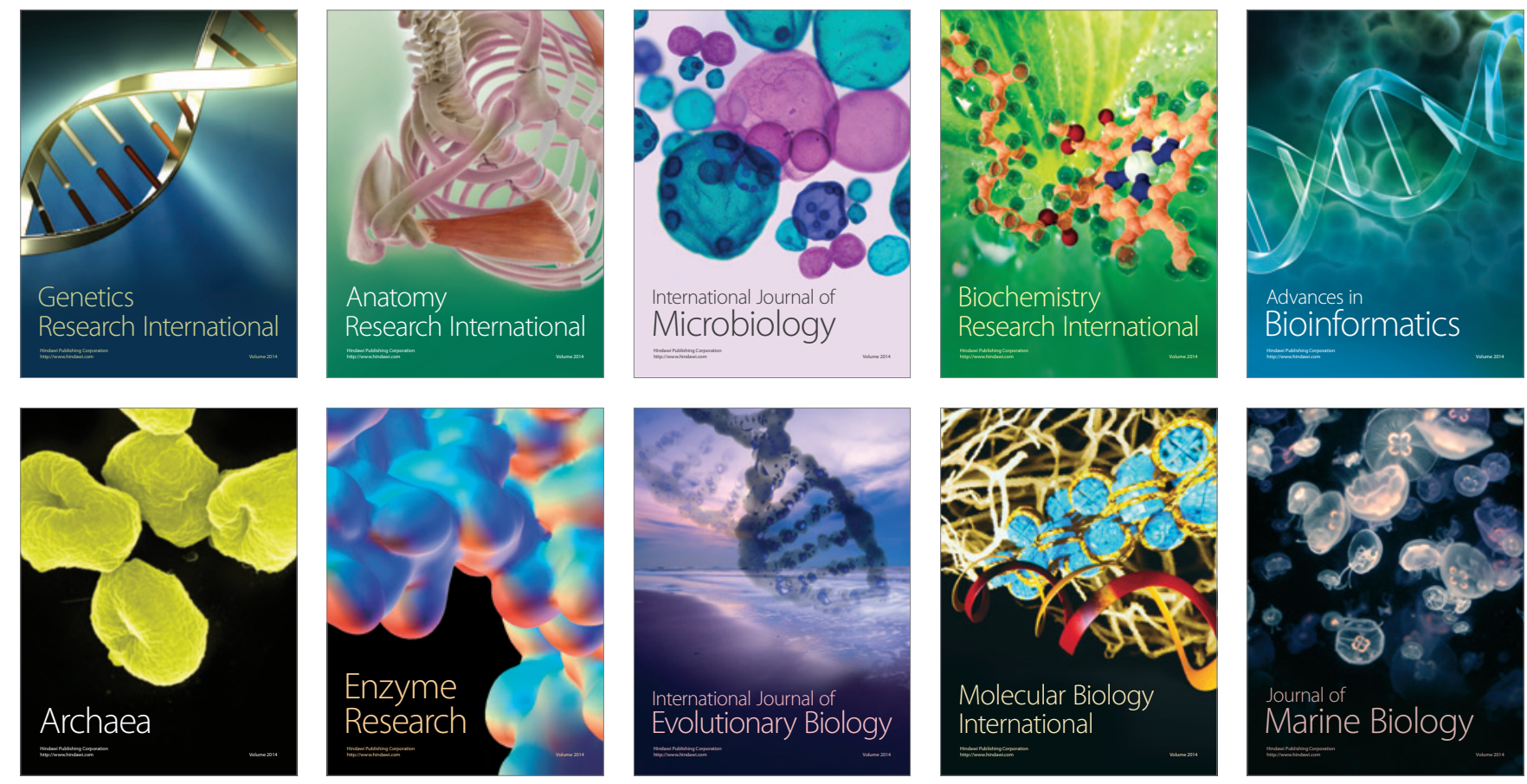